\title{
Grußwort
}

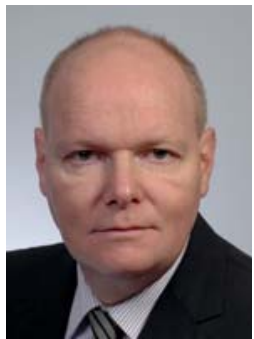

Liebe DMV-Mitglieder,

die öffentliche Diskussion um bildungspolitische Fragen ist in vollem Gang - geht es um das enttäuschende Abschneiden der deutschen Grundschülerinnen und -schüler in der neuesten TIMSS-Studie, die Digitalkompetenz, die deutscher Schulunterricht zur Vorbereitung auf die Arbeitswelt von morgen vermitteln soll oder die Realisierung eines bundesweiten Zentralabiturs in Mathematik. Die DMV ist in diesen Diskussionen eine gefragte Partnerin, die insbesondere inhaltliche Aspekte kompetent repräsentiert und für ein hohes fachliches Niveau eintritt. Es ist klar, dass die Ergebnisse dieser heute geführten Diskussionen die Weichen für morgen und auch übermorgen stellen. Deshalb ist Ihre Beteiligung, liebe Mitglieder, durch Meinungsäußerungen und Diskussionbeiträge besonders wichtig. Die Pressemitteilung der DMV zur Digitalisierungsstrategie des BMBF von Mitte November 2016 (siehe folgende Seite), die viel Zustimmung aber auch viel Widerspruch erfahren hat, ist dafür ein schönes Beispiel.

Auch der in diesem Heft veröffentlichte, interessante Artikel „Statistiken zum Mathematikstudium“ von Berndtsen, Dieter und Törner zu den Anfängerzahlen wird mit seinen Thesen (hoffentlich) eine Diskussion unter den Lesern der MDMV initiieren. Schon jetzt steht fest, dass das durch DESTATIS bereitgestellte Datenmaterial von heterogener Qualität ist und in einigen Bundesländern zu systematischen Fehlbilanzen führt. Mit Blick auf die Tatsache, dass die Daten von DESTATIS auch als offizielle Quelle zur Implementierung politischer Entscheidungen herangezogen werden und insofern die Realität definieren, ist es die Aufgabe der DMV, diese Daten und Ihre Erhebungsweise im Auge zu behalten.

Mit dem Jahresende 2016 endet auch meine Amtszeit als Präsident der DMV, und dies gibt mir Gelegenheit für einen Rückblick. In diese Zeit fielen zwei schöne Jahrestagungen 2015 in Hamburg und 2016 gemeinsam mit der GAMM in Braunschweig. Schon wegen der großen Teilnahme von über 850 bzw. über I 300 Mathematikerinnen und Mathematikern werte ich diese als Erfolg und hoffe auf eine Fortsetzung dieses Trends bei der nächsten Jahrestagung im September 2017 in Salzburg gemeinsam mit der ÖMG. Auch die Gauß-Vorlesungen 2015 in Stuttgart und in Münster und 2016 in Dresden waren jeweils ein voller Erfolg und eine wunderbare Werbung für Mathematik und die DMV - ich freue mich schon auf die GaußVorlesung am 19. Januar 2017 an der TU München! Ebenso erfolgreich und würdevoll waren die Auszeichnungen von Norbert Henze und von Ilja Agricola mit dem Ars legendi-Fakultätenpreis für exzellente Lehre in der Mathematik 2015 bzw. 2016 sowie von Moritz Epple und seinem Team mit dem DMV-Medienpreis 2015 und von Veronika Mischitz mit dem DMV-Journalistenpreis 2015.

Neben diesen gut sichtbaren Aktivitäten hat das von mir geleitete DMV-Präsidium viele wichtige Schritte zur Weiterentwicklung der DMV gemacht, die man von außen erst auf den zweiten Blick bemerkt. Im Sommer 2016 ist Frau Kirstein-Gaekel zu Frau Jahnke in die Geschäftsstelle gekommen und wird bis zum Eintritt Frau Jahnkes in den Ruhestand die Aufgaben Schritt für Schritt übernehmen. Ich danke Frau Jahnke für die ausgezeichnete Zusammenarbeit und wünsche ihr ebenso wie Frau KirsteinGaekel alles Gute für die Zukunft! Im Sommer 2016 wurde auch der mathematische Adventskalender Mathe im Advent (MiA) aus der DMV ausgegliedert und wird seither von einer gleichnamigen gemeinnützigen $\mathrm{GmbH}$ realisiert - unter der bewährten Leitung von Stephanie Schiemann und Robert Wöstenfeld. Die Notwendigkeit dieses Schritts ergab sich paradoxerweise aus dem überwältigenden Erfolg von MiA, zu dessen Betrieb Finanzmittel in einer Höhe erforderlich sind, die im Vergleich zum Jahresbudget der DMV keine kleine Zahl mehr ist. Ich wünsche Frau Schiemann und Herrn Wöstenfeld, sicher auch in Ihrem Namen, liebe Mitglieder, viel Erfolg!

Für ihre bildungspolitische Platzierung ist die Abstimmung der DMV mit ihren Schwestergesellschaften GDM und MNU in der gemeinsamen Kommission zum Übergang Schule-Hochschule sehr wichtig. Hier werden beispielsweise die zu Anfang genannten politischen Diskussionsthemen aufgegriffen und vertieft. Politisch gleichermaßen bedeutsam sind die seit nurmehr drei Jahren halbjährlich stattfindenden Treffen der Verbandsspitzen von DMV, DPG, GDCh, VBio und DVGeo. Hier stimmen sich die beteiligten Gesellschaften zu wissenschaftspolitischen Themen ab, wie Hochschulranking oder Open Access.

Die DMV behält dabei ihr eigenes Profil und ihren in der Satzung festgelegten Zweck im Blick. So trat die DMV zum Ende 2015 aus der ASIIN aus, im März 2016 drückte die DMV ihre Besorgnis über die Entwicklung in der Türkei gemeinsam mit der DVMLG und dem DVGeo in einem Brief an den türkischen Ministerpräsidenten aus, im Mai 2016 begrüßte die DMV den COSHMindestanforderungskatalog und empfahl ihn Studienanfängern in MINT-Fächern zur Orientierung, und vor kurzem hat die DMV kritisch zur anfangs erwähnten Digitalisierungsstrategie des BMBF Stellung bezogen.

Ohne die Mithilfe aller Präsidiumsmitglieder und meines Vorgängers im Amt, Jürg Kramer, sowie der exzellenten Zuarbeit von Geschäftsstelle, des Medienbüros 
mit Thomas Vogt und dem neu hinzugekommenen David Vogel, des Netzwerkbüros mit Stephanie Schiemann, meiner Sekretariatskraft Ingrid Stoll und des MDMVRedaktionteams mit Christoph Eyrich und Antje Schulze wäre die Führung der DMV schlichtweg nicht möglich gewesen - ihnen allen danke ich herzlich und wünsche meinem Nachfolger Michael Röckner und dem neuen Vizepräsidenten Friedrich Götze alles Gute für ihre Amtszeit!

Ihr Volker Bach

\section{Pressemitteilung der DMV zur „Bildungsoffensive zur digitalen Wissensgesellschaft“}

Zum Nationalen IT-Gipfel, der am 16. und 17. II.2016 in Saarbrücken stattfindet, appelliert die Deutsche Mathematiker-Vereinigung: Nicht das bloße Verwenden digitaler Medien, sondern das Verständnis ihrer Grundlagen schafft die Voraussetzung für einen souveränen digitalen Wandel. Ohne die richtigen Lerninhalte bleiben Soft- und Hardwarelieferungen eine Scheinlösung.

Berlin, 22. November 2016. Der Vorstand der Deutschen Mathematiker-Vereinigung fasst seine Position zur „Bildungsoffensive zur digitalen Wissensgesellschaft“ wie folgt zusammen.

Um die technischen, wirtschaftlichen und gesellschaftlichen Herausforderungen der Digitalisierung zu meistern, muss auf allen Ebenen der Bildung gehandelt werden. Wir begrüßen den Willen der deutschen Bildungspolitik, ihren Handlungsspielraum auf diesem Feld zu nutzen. Mit ihrer gegenwärtigen Schwerpunktsetzung schafft die „Bildungsoffensive zur digitalen Wissensgesellschaft" aus unserer Sicht allerdings eine Scheinlösung und könnte ihr Ziel verfehlen.

Deshalb plädieren wir dafür, den Schwerpunkt der Debatte und der „Offensive“ zu verschieben. Ziel sollte sein, grundlegende Kompetenzen zu vermitteln, die Lernende zu einem mündigen Umgang mit digitalen Neuerungen befähigen. Wir fordern: Inhalte statt Geräte!

Tablets und soziale Netzwerke verwenden zu können, heißt noch lange nicht, den digitalen Wandel zu meistern. Vollständig digital kompetent ist und bleibt auf lange Sicht nur, wer die theoretischen Grundlagen versteht. Diese Grundlagen entstehen nicht als Nebeneffekt beim Lernen mit digitalen Medien, sondern müssen separat und fokussiert im Unterricht vermittelt werden. Digitale Medien können und sollten hierzu nur ergänzend eingesetzt werden. Nach Erfahrung der überwältigenden Mehrheit der Mathematikerinnen und Mathematiker weltweit sind Tafel, Papier und das direkte Unterrichtsgespräch meist viel besser geeignet. Auch dürfen diese Grundlagen nicht al- lein Hochbegabten oder digital Affinen vorbehalten bleiben, sondern müssen Teil der Allgemeinbildung werden.

Ohne die vorherige Vermittlung dieser Grundlagen ist die Belieferung von Bildungseinrichtungen mit Soft- und Hardware hingegen eine Scheinlösung. Bleiben die richtigen Lerninhalte aus, hemmt sie sogar den Anstieg der Digitalisierungskompetenz in Deutschland. Stattdessen wird die Lernzeit der Lernenden überfrachtet, und oberflächlich sinnvolle Inhalte verdrängen den Erwerb von Grundlagen.

Wir halten es daher für fehlgeleitet, an erster Stelle in digitale Medien zu investieren. Reduziert man Digitalisierungskompetenz auf den Umgang mit digitalen Medien, so glaubt man irrig, Digitalisierungskompetenz entstehe erschöpfend im Lernen mithilfe digitaler Medien. Das Gegenteil ist aber richtig: Erst die auf Grundlagen sorgfältig aufbauende Digitalkompetenz kann das enorme Potenzial moderner Hard- und Software voll nutzen! Digitale Medien sollten nicht um ihrer selbst willen und auf Kosten der Zukunft Lernender gefördert werden.

Wir fordern über die föderalen Hemmnisse hinweg, die allgemeinen Rahmenbedingungen für die Bildung zu verbessern. Wir fordern den Unterricht in den Fächern Mathematik und Informatik zu stärken und nicht weiter auszudünnen, damit hier die grundlegende und langfristige Digitalisierungskompetenz vermittelt werden kann. Wir fordern, die Lehrerbildung in diesen Bereichen substanziell zu verbessern. Wir fordern, analytisches Denken gezielt zu lehren und diesem Anliegen Priorität zu geben.

\section{Pressekontakt}

David Vogel und Thomas Vogt

Medienbüro Mathematik

Freie Universität Berlin

Institut für Mathematik

Arnimallee 7

14195 Berlin

Tel. (030) 83875657

medienbuero@mathematik.de

www.dmv.mathematik.de 